\title{
Tetranuclear Dysprosium Single-Molecule Magnets: Tunable Magnetic Interactions and Magnetization Dynamics through Modifying Coordination Number
}

\author{
Kun Zhang, ${ }^{\text {ab }}$ Gao-Peng Li, ${ }^{b}$ Vincent Montigaud, ${ }^{d}$ Olivier Cador, ${ }^{d}$ Boris Le Guennic, ${ }^{*, d}$ Jinkui Tang $^{*, c}$ and Yao-Yu Wang ${ }^{*, b}$
}

The study of mononuclear lanthanide-based systems, where the observed Single Molecule Magnets (SMMs) properties originate from the local magnetic anisotropy of the single lanthanide ion, has been extensively investigated in the literature. The case for polynuclear lanthanide SMMs becomes more challenging both experimentally and theoretically due to the complexity of such architectures involving interactions between the magnetic centers. Much interest was devoted to the study of the structural effect on the magnetic interactions and relaxation dynamics. However, the understanding of the structural influence on those two factors remains a difficult task. To address this issue, a system containing two structurally related tetranuclear Dy(III) SMMs, namely $\left.\mathrm{Dy}_{4}(\mathrm{~L})_{4}(\mathrm{OH})_{2}(\mathrm{DMF})_{4}\left(\mathrm{NO}_{3}\right)_{2}\right] \cdot 2(\mathrm{DMF}) \cdot\left(\mathrm{H}_{2} \mathrm{O}\right)$ (1) and $\left[\mathrm{Dy}{ }_{4}(\mathrm{~L})_{4}(\mathrm{OH})_{2}\left(\mathrm{DMF}_{2}\left(\mathrm{NO}_{3}\right)_{2}\right](\mathbf{2}) \quad\left(\mathrm{H}_{2} \mathrm{~L}=2-\left(2-\mathrm{hydroxy}_{-3}-\right.\right.\right.$ methoxybenzylideneamino)phenol), is introduced and investigated. Through modifying the ligands on the changeable coordination sites, the intramolecular magnetic interactions and relaxation dynamics in these two Dy(III) $)_{4}$ SMMs can be tuned. Both complexes exhibit slow relaxation of their magnetization with a relaxation barrier of $114 \mathrm{~K}$ for complex 2 while a blocking temperature below $2 \mathrm{~K}$ is observed for complex 1 . Ab initio calculations reveal that changes in coordination numbers and geometries on the Dy(III) sites can significantly affect the magnetic interactions as well as single-ion anisotropy. The combination of experimental work and $a b$ initio calculations offers insight into the relationship between structures and magnetic properties and sheds light on the rational design of future polynuclear lanthanide SMMs with enhanced magnetic properties.

\section{Introduction}

Considerable attention has been bestowed on single molecule magnets (SMMs) due to their great potential applications in molecular spintronics, quantum computing and high-density information storage. ${ }^{1-5}$ These molecular systems are characterized by an intrinsic magnetic anisotropy and slow relaxation of their magnetization. ${ }^{6}$ The lanthanide Dy(III) ion, by virtue of its intrinsic large magnetic anisotropy and Kramers doublet ground state of ${ }^{6} \mathrm{H}_{15 / 2}$, has shown to be the most appealing constituent for the construction of SMMs with high

\footnotetext{
a. School of Textile Science and Engineering, Xi'an Polytechnic University, Xi'an 710048, P.R. China. E-mail: zhangkun625@foxmail.com.

${ }^{b}$ Key Laboratory of Synthetic and Natural Functional Molecule Chemistry of the Ministry of Education, Shaanxi Key Laboratory of Physico-Inorganic Chemistry, College of Chemistry \& Materials Science, Northwest University, Xi'an, 710127, P.R. China.E-mail: wyaoyu@nwu.edu.cn.

c. State Key Laboratory of Rare Earth Resource Utilization, Changchun Institute of Applied Chemistry, Chinese Academy of Sciences, Changchun, 130022, P.R. China. E-mail: tang@ciac.ac.cn.

d. Univ Rennes, CNRS, ISCR (Institut des Sciences Chimiques de Rennes) - UMR 6226, F-35000 Rennes, France.E-mail: boris.leguennic@univ-rennes1.fr.

K. Zhang and G.-P. Li contributed equally to this work.

Electronic Supplementary Information (ESI) available: Additional magnetic data, additional figures. CCDC 1884388 for 2. For ESI and crystallographic data in CIF or other electronic format see DOI: 10.1039/x0xx00000x
}

performance, which has been demonstrated by the dysprosium metallocene cation $\left[\left(C p^{i P r 5}\right) \operatorname{Dy}\left(C p^{*}\right)\right]^{+}\left(C p^{i P r 5}=\right.$ penta -iso-propylcyclopentadienyl, $\mathrm{Cp}^{*}=$ pentamethylcyclopentadien yl) with record magnetic hysteresis at temperatures of up to $80 \mathrm{~K}$ and relaxation barrier of $1541 \mathrm{~cm}^{-1}$. Therefore, interest towards developing Dy(III) SMMs has been continuously growing. In this research activity, subsequent endeavors are increasingly devoted to the understanding of magnetostructural relationships, which could in turn offers guidelines for the structural optimization of magnetic properties. ${ }^{8-12}$ In fact, achievements have been made to illustrate how the structural and electrostatic perturbation affects the relaxation dynamics in mononuclear Dy(III) SMMs through modulating the coordination environment around metal centers combined with ab initio calculation analysis. ${ }^{13-16}$ According to those explorations, outstanding mononuclear Dy(III) SMMs can be reached by synthesizing the architectures with high-symmetry geometry and/or strong axial crystal field. ${ }^{17-24}$ However, for systems containing two or more metal ions, the case becomes more challenging both experimentally and theoretically due to the complexity of such architectures involving interactions between the magnetic centers, ${ }^{25-28}$ for which the magnetic phenomena become hardly predictable. ${ }^{29-34}$ Even so, understanding the relationship between structure and magnetic anisotropy involving single metal ion as well as 
magnetic interactions between two ions becomes more important when such properties have been shown to synergically improve the magnetic relaxation properties in polynuclear Dy(III) molecules. ${ }^{35-37}$

Recently, it has been demonstrated that even subtle variations of the coordination environment around Dy(III) ion might drastically change the single-ion magnetic anisotropy and magnetic interactions involved in polynuclear Dy(III) SMMs. 33,34 Therefore, an approach based on minute changes to the structure and/or electronic properties becomes an effective strategy to explore the factors influencing the magnetic interactions and relaxation dynamics, which would led to advances in the design of higher performance polynuclear Dy(III) SMMs. ${ }^{37,38}$ However, due to the variable and high coordination numbers as well as poor directionality of the rare-earth metal, modulating the coordination environment (coordination numbers and geometries) around metal centers in polynuclear Dy(III) architectures is very difficult. ${ }^{39}$ Thus, a current challenge is to assemble targeted polynuclear dysprosium(III) SMMs, thereby providing a means of tuning the relaxation dynamics and magnetic interactions through the influence of different coordination environments around the metal site.

Herein, we demonstrate an intriguing and promising route to modulate the magnetic interactions and relaxation dynamics in Dy(III) ${ }_{4} \mathrm{SMMs}^{40}$ Specifically, the template complex $\left[\mathrm{Dy}_{4}(\mathrm{~L})_{4}(\mathrm{OH})_{2}(\mathrm{DMF})_{4}\left(\mathrm{NO}_{3}\right)_{2}\right] \cdot 2(\mathrm{DMF}) \cdot\left(\mathrm{H}_{2} \mathrm{O}\right)\left(\mathbf{1} ; \mathrm{H}_{2} \mathrm{~L}=\right.$ 2-(2-hydroxy3-methoxybenzylideneamino)phenol), reported in our previous work, possesses two equivalent changeable Dy(III) sites with tunable coordination environment. ${ }^{34}$ In the present work, we designed a Dy $(\mathrm{III})_{4} \mathrm{SMM}\left[\mathrm{Dy}_{4}(\mathrm{~L})_{4}(\mathrm{OH})_{2}(\mathrm{DMF})_{2}\left(\mathrm{NO}_{3}\right)_{2}\right](\mathbf{2}$, Figure 1), by removing two DMF ligands at the Dy1 site compared to complex $\mathbf{1}$, while the coordination environment around Dy2 center is not changed. Through this targeted modification of coordination number and geometry on determined metal sites, the intramolecular magnetic interactions and relaxation dynamics in these two Dy $(\mathrm{III})_{4}$ SMMs can be modulated. Thus, the present [Dy $y_{4}$ system provides an elegant model to understand the factors that influence the magnetic interactions and relaxation dynamics and a simple way to optimize the magnetic performance of the polynuclear dysprosium SMMs.

\section{Experimental section}

\section{Chemicals and Physical Measurements}

All reagents were purchased from commercial sources and used as received. 2-(2-Hydroxy-3-methoxybenzylideneamino)phenol $\left(\mathrm{H}_{2} \mathrm{~L}\right)$ was prepared by condensation of 2-aminophenol and o-vanillin in a 1:1 molar ratio in hot ethanol according to a modified procedure reported previously. ${ }^{41}$ All reactions were carried out under aerobic conditions. Elemental analyses $(\mathrm{C}, \mathrm{H}, \mathrm{N})$ were determined with an Elementar vario EL III Analyzer. The FT-IR spectra were recorded from $\mathrm{KBr}$ pellets in the range $4000-400 \mathrm{~cm}^{-1}$ on a Bruker Tensor 27 spectrometer. The magnetization data of complex 2 was recorded on a Quantum Design VSM SQUID magnetometer equipped with a 7
T magnet. Variable-temperature magnetization was measured with an external magnetic field of $500 \mathrm{Oe}$ in the temperature range of 1.8-300 $\mathrm{K}$ and the frequency dependent ac susceptibility was measured with an oscillating field of 2.0 Oe. Finely ground microcrystalline powder of $\mathbf{2}$ was immobilized in eicosane matrix inside a polycarbonate capsule. Both the contributions of the eicosan and the capsule have been subtracted from the data obtained. Phase purity was checked by powder X-ray diffraction (PXRD) using a Bruker AXS D8 Advance diffractometer with Cu-Ka ( $\lambda$ $=1.54056 \AA$ A) radiation.

Synthesis of Complex $\left[\mathrm{Dy}_{4}(\mathrm{~L})_{4}(\mathrm{OH})_{2}(\mathrm{DMF})_{2}\left(\mathrm{NO}_{3}\right)_{2}\right]$ (2) The mixture of Schiff-base $\mathrm{H}_{2} \mathrm{~L}$ ligand $(0.2 \mathrm{mmol}, 48.6 \mathrm{mg})$ and triethylamine $(0.4 \mathrm{mmol}, 0.06 \mathrm{ml})$ in acetonitrile $\left(\mathrm{CH}_{3} \mathrm{CN}\right)(5 \mathrm{ml})$ was stirred for $10 \mathrm{~min}$. Then, solid Dy $\left(\mathrm{NO}_{3}\right)_{3} \bullet 6 \mathrm{H}_{2} \mathrm{O}(0.3 \mathrm{mmol}, 131.6 \mathrm{mg})$ was added and further stirred for $30 \mathrm{~min}$. Next, after stirring for 30 min, the dimethylformamide (DMF) $(5 \mathrm{ml})$ solution of hexafluoroacetylacetone $(0.2 \mathrm{mmol}, 0.03 \mathrm{ml})$ and triethylamine $(0.2$ $\mathrm{mmol}, 0.03 \mathrm{ml}$ ) was added to the above mixture and further stirred for $1 \mathrm{~h}$. The resulting solution was filtered and the filtrate was allowed to stand at room temperature for two days. Yellow blockshaped crystals suitable for single crystal analysis were formed by slow evaporation of solvent and were collected by careful filtration (58\% yield based on Dy). IR spectra $\left(\mathrm{cm}^{-1}\right)$ : $3612(\mathrm{~m}), 3421(\mathrm{~m})$, 3057(m),2999(m), 2935(m), 1645(s), 1604(s), 1549(m), 1483(s), $1458(\mathrm{~s}), 1383(\mathrm{~s}), 1281(\mathrm{~s}), 1252(\mathrm{~s}), 1223(\mathrm{~s}), 1174(\mathrm{~m}), 1101(\mathrm{~m})$, 1072(m), 1030(m), 970(m), 866(m), 820(m), 739(m), 700(m), $634(\mathrm{w}), 567(\mathrm{~m}), 503(\mathrm{~m})$. Elemental analysis found (calc.)\% for complex 2: C: 38.69(38.80); H: 3.09(3.15); N:5.88(5.84).

\section{X-Ray Crystallography}

Single-crystal X-ray diffraction measurements of the complexes were carried out on a Bruker Apex II CCD diffractometer with graphite monochromated MoK $\alpha$ radiation $(\lambda=0.71073 \AA$ ) ) at $296 \mathrm{~K}$ for 2. The structures were solved using the direct method (SHELXS) and refined by means of the full-matrix least-squares method (SHELXL) on $\mathrm{F}^{2} .^{42}$ Anisotropic thermal parameters were used for the non-hydrogen atoms and isotropic parameters for the hydrogen atoms. Hydrogen atoms were added geometrically and refined using a riding model. Crystallographic data and refinement details are given in Table S1. CCDC 1884388 (2) contains the supplementary crystallographic data for this paper.

\section{Results and discussion}

Crystal structures

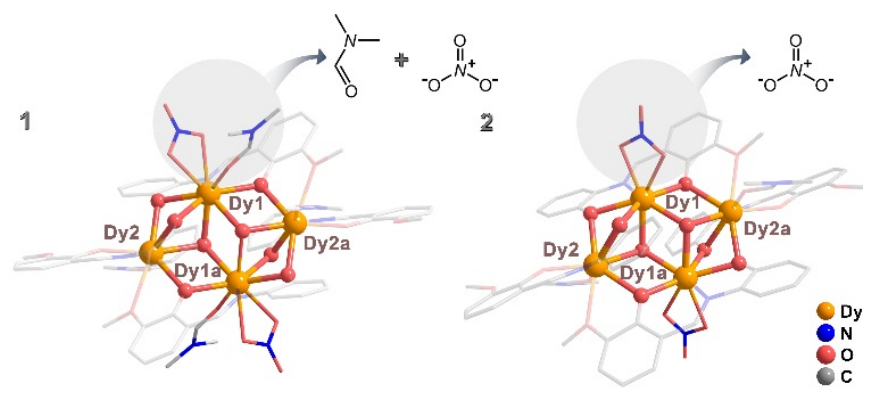

Fig. 1 Molecular structure of $\mathbf{1}$ and $\mathbf{2}$, hydrogen atoms and solvent molecules have been omitted for clarity. The $\left\{\mathrm{Dy}_{4} \mathrm{O}_{8}\right\}$ core and the 
modification of coordination environment around Dy1 and Dy1a were highlighted.

Single-crystal X-ray structural analysis reveals that the two complexes are centrosymmetric and crystallize in the monoclinic $P 2_{1} / c$ space group. As seen in Fig. 1 , the key feature of both complexes is that four Dy(III) ions are nearly coplanar in a regular parallelogram, forming a distorted defective dicubane $\left\{\mathrm{Dy}_{4} \mathrm{O}_{8}\right\}$ core. Specifically, in each of two symmetry-related $\mathrm{Dy}_{2}(\mathrm{~L})_{2}$ moieties of both complexes, two Dy(III) ions (Dy1 and Dy2 or Dy1a and Dy2a) are linked by two $\mu_{2}$-phenoxo oxygen atoms from two symmetry equivalent $\mathrm{L}^{2-}$ ligands, and the moieties are further linked by two $\mu_{3^{-}}$ hydroxide ligands. Only small structural variations for the geometry of $\left\{\mathrm{Dy}_{4} \mathrm{O}_{8}\right\}$ core were found in the two complexes (Supporting Information, Table S2). The coordination environment of Dy2 in both complexes is completed by one $\mathrm{N}$ atom and four phenoxo $\mathrm{O}$ atoms from $\mathrm{L}^{2-}$ ligands and one $\mu_{3}$-hydroxide $\mathrm{O}$, one methoxide $\mathrm{O}$ and one DMF $\mathrm{O}$, making it eight-coordinated with a distorted square antiprism geometry. The very similar continuous shape measures (5.413 and 6.206 for $\mathbf{1}$ and 2, respectively) of Dy2 determined by SHAPE software ${ }^{43}$ and bond lengths and angles (Table S3) reveal almost identical coordination spheres of the Dy2 centers in $\mathbf{1}$ and $\mathbf{2}$. The Dy $\mathbf{1}$ in $\mathbf{1}$ is nine-coordinated and builded with one $\mathrm{N}$ atom and three phenoxo $\mathrm{O}$ atoms from $\mathrm{L}^{2-}$ ligands and two $\mu_{3}$ - hydroxide $\mathrm{O}$, two nitrate $\mathrm{O}$ and one DMF $\mathrm{O}$, adopting a distorted tricapped trigonal prism with a $D_{3 \mathrm{~h}}$ point group. In contrast, the Dy1 coordination sphere in complex $\mathbf{2}$ is obtained by removing the DMF ligand from the Dy1 in complex 1 (Fig. 1). Consequently, the Dy1 became eight-coordinated surrounded by one $\mathrm{N}$ atom and three phenoxo $\mathrm{O}$ atoms from $\mathrm{L}^{2-}$ ligands and two $\mu_{3}$ - hydroxide 0 , two nitrate 0 , adopting a distorted biaugmented trigonal prism with a $C_{2 v}$ point group. In addition, the intermolecular Dy•••Dy distances are over $9 \AA$.

\section{Magnetic properties}

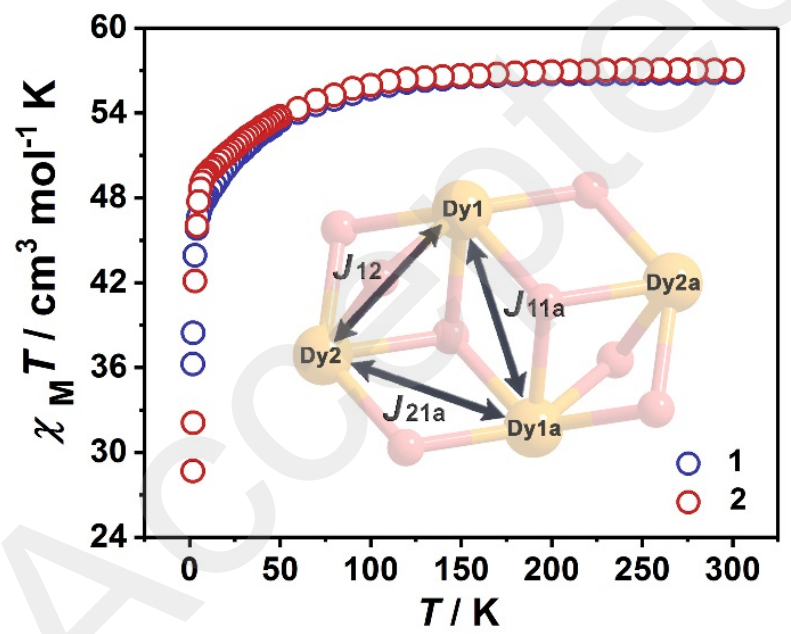

Fig. 2 Plots of the $\chi_{M} T$ versus temperature for complexes $\mathbf{1}$ and $\mathbf{2}$ in an applied magnetic field of 500 Oe. Inset: schematic representation of the contributions of magnetic interactions considered in the calculations.

Direct current magnetic susceptibility measurements of polycrystalline samples of complexes $\mathbf{1}$ and $\mathbf{2}$ are performed under an external field of $500 \mathrm{Oe}$ in the temperature range of $1.8-300 \mathrm{~K}$ to probe their magnetic behavior (Fig. 2). The room-temperature $\chi_{\mathrm{M}} T$ values of 56.86 and $57.04 \mathrm{~cm}^{3} \mathrm{~K} \mathrm{~mol}^{-1}$ for 1 and 2 are in good agreement with the expected value of $56.68 \mathrm{~cm}^{3} \mathrm{~K} \mathrm{~mol}^{-1}$ for four uncoupled Dy(III) $\left({ }^{6} \mathrm{H}_{15 / 2}, g={ }^{4} / 3\right)$ ions. ${ }^{44}$ When decreasing the temperature, both complexes behave similarly with a decrease of the $\chi_{\mathrm{M}} T$ product. A sharp decrease below $50 \mathrm{~K}$ is observed upon lowering temperature, reaching minimum values at $1.8 \mathrm{~K}$ of 36.26 $\mathrm{cm}^{3} \mathrm{~K} \mathrm{~mol}^{-1}$ for $\mathbf{1}$ and 28.69 for 2 . The observed decrease of $\chi_{\mathrm{M}} T$ can be attributed to the gradually thermal depopulation of the Stark sublevels of the Dy(III) ions and/or intramolecular antiferromagnetic magnetic interaction between the Dy(III) ions, as observed in other dysprosium complexes. ${ }^{45}$

The magnetization (M) plots as a function of field $(H)$ for complex 2 below $5 \mathrm{~K}$ (Fig. S2) reveals a relatively rapid increase of the magnetization at low fields followed by a slow linear increase at high fields without a clear complete saturation. The high-field linear variation of the magnetization suggests the presence of excited states relatively closed in energy to be mixed by the magnetic field. This is also supported by the observation that, while plotting the $M$ vs $\mathrm{HT}^{-1}$ at different fields (Fig. S2), the curves are not all superimposed on a single master-curve. ${ }^{46}$ As already showed for $\mathbf{1}$, no hysteresis was observed for 2 , in the range of $[-30 ; 30] \mathrm{kOe}$ at $1.8 \mathrm{~K}$ (Fig. S3).

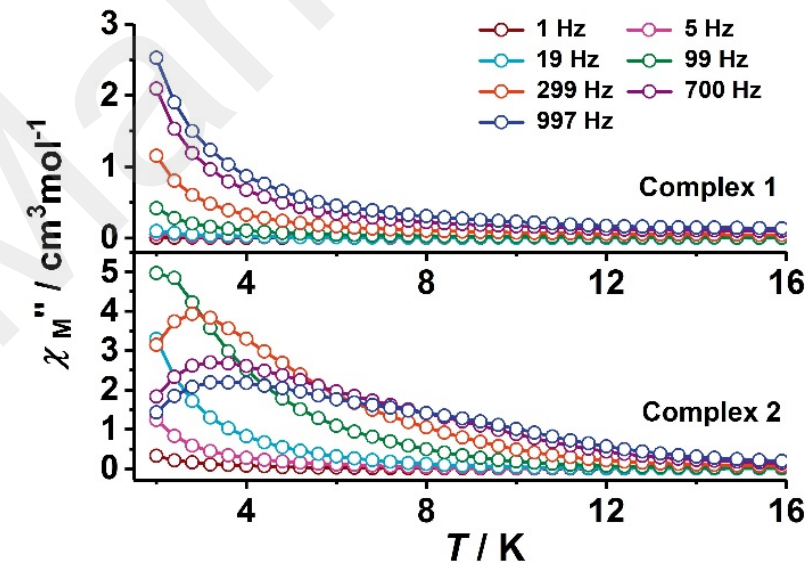

Fig. 3 Comparison of temperature dependent out-of-phase $\left(\chi_{M}{ }^{\prime \prime}\right)$ ac susceptibility for complexes $\mathbf{1}$ and $\mathbf{2}$ under zero dc field.

Compared to static magnetic properties, the dynamic properties of lanthanide SMMs appear to be more sensitive to a subtle change of the coordination environment surrounding the Dy(III) centers (in our case, the coordination number and geometry), which can be detected through ac magnetic susceptibility measurements at low temperature. ${ }^{47}$ As shown in Fig. 3 and $\mathrm{S} 4$, both complexes display temperature-dependent $\chi_{\mathrm{M}}$ " signal, which indicates slow relaxation of the magnetization. However, no maximum value was observed above $2 \mathrm{~K}$ for complex 1 , suggesting a blocking temperature lower than $2 \mathrm{~K}$. In contrast, complex 2 shows different behaviour. The maximum of $\chi_{\mathrm{M}}{ }^{\prime}$ and $\chi_{\mathrm{M}}{ }^{\prime \prime}$ value at $997 \mathrm{~Hz}$ are much higher than that of complex 1 (Fig. 3). In order to extract relaxation times $(\tau)$, ac susceptibility data can be fitted with a generalized Debye model, as shown in Cole-Cole $\left(\chi_{\mathrm{M}}{ }^{\prime \prime}\right.$ vs. $\left.\chi_{\mathrm{M}}{ }^{\prime}\right)$ diagrams (Fig. S5, S6, Table S4). ${ }^{48}$ The plot of $\ln \tau$ versus $T^{-1}$ was obtained to gain insight into the dynamics of relaxation of the magnetization of $\mathbf{2}$. As seen in Fig. 4, an obvious curvature showed in the plot, indicating that a 
combination of other relaxation mechanisms has to be taken into account. It is worth noting that other relaxation processes, such as Raman process, can coexist with Orbach relaxation. Therefore, the data over the entire temperature range were analyzed by the following equation (1):

$$
\ln \tau=-\ln \left[C T^{n}+\tau_{0}^{-1} \exp \left(-U_{\text {eff }} / k_{\mathrm{B}} T\right)\right]
$$

where the two terms represent the Raman and Orbach processes, respectively ( $C$ is the coefficient of Raman process, $U_{\text {eff }}$ is the energy barrier to magnetization reversal, and $k_{\mathrm{B}}$ is the Boltzmann constant). The best fitting was found for $C=162.5 \mathrm{~s}^{-1} \mathrm{~K}^{-1.6}, \mathrm{n}=1.6, \tau_{0}=1.58 \times$ $10^{-9} \mathrm{~s}$, and $U_{\text {eff }} / k_{\mathrm{B}}=114 \mathrm{~K}$. The obtained $\tau_{0}$ value falls into the expected range of $10^{-5}-10^{-12} \mathrm{~s}$ for the Dy(III)-based SMMs reported previously. ${ }^{49}$

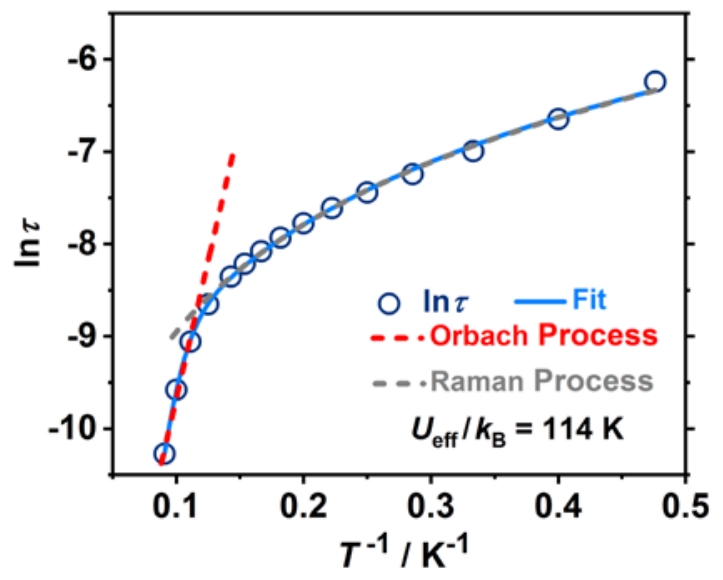

Fig. 4 Plot of $\ln (\tau)$ versus $T^{-1}$ for $\mathbf{2}$ under zero dc-field, where $\tau$ is the relaxation time, $T$ is the temperature (K). The blue line represents the fit to multiple relaxation processes using eqn 1.

\section{Theoretical Calculations}

To give more insights in the influence of the Dy(III) coordination sphere modifications on the single-ion magnetic properties as well as on the intramolecular magnetic interactions in these complexes, $a b$-initio SA-CASSCF/SI-SO calculations were performed using the Molcas 8.0 program package (computational details in Supporting Information). ${ }^{50}$ Following the computational protocol employed in our previous work on a model complex $\mathbf{1}^{\prime},{ }^{34}$ the principal components of the $g$ tensor and the wave function composition of the ground state multiplet are calculated for each Dy centers of the model complex $2^{\prime}$, in the effective spin $1 / 2$ approximation. The orientations of the main $g$-values ( $g_{z}$ components) of each Dy center are represented in Fig. 5.
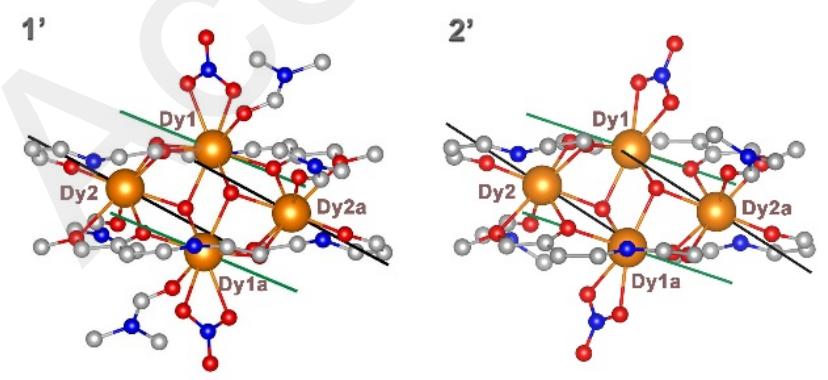

Fig. 5 Schematic representation of the main $\mathrm{g}$ tensor component of each Dy center, green line for Dy1 and Dy1a and black line for Dy2 and Dy2a, for the model complexes $\mathbf{1}^{\prime 34}$ and $\mathbf{2}^{\prime}$. Dy, O, N, F and C atoms are represented as orange, red, blue, yellow and grey spheres, respectively. $\mathrm{H}$ atoms are omitted for clarity.

The results revealed almost identical features at the Dy2/Dy2a sites between the two complexes with an almost pure (97\%) $| \pm 15 / 2\rangle$ ground state and an Ising type, strongly axial anisotropy $\left(\mathrm{g}_{z}\right.$ around 19.7), consistent with the above structural analysis (Table S5-S8). For Dy1/Dy1a (the metal centers with varying coordination numbers and geometries, vide supra), a different behavior was found between model complexes $\mathbf{1}^{\prime}$ and $\mathbf{2}^{\prime}$. Indeed, a mixed ground state with a major contribution of the $| \pm 15 / 2\rangle$ spin states was obtained for Dy1 of 1' $(77 \%| \pm 15 / 2>+15 \%| \pm 11 / 2>)$ corresponding to a calculated ground state $g$ tensor $\left(g_{x}=0.49, g_{\gamma}=1.22, g_{z}=18.06\right)$. In 2', the variation of the coordination sphere around the Dy1/Dy1a centers led to a decrease of the transversal components of the $g$ tensor $\left(\mathrm{g}_{\mathrm{X}}=0.17, \mathrm{~g}_{\mathrm{Y}}=0.46\right.$ and $\left.\mathrm{g}_{\mathrm{Z}}=18.45\right)$ with a mixed ground state $(80 \%| \pm 15 / 2>+14 \%| \pm 11 / 2>)$.

In order to get a global picture of the magnetic properties observed in these complexes, intramolecular interactions (Fig. 2) are taken into account in the calculations while intermolecular magnetic interactions are not considered due to the large intermolecular Dy $\cdots$ Dy distances. The representation of the calculated magnetic susceptibility for model $2^{\prime}, \chi_{\mathrm{M}} T$ in the temperature range of $2-300 \mathrm{~K}$ are presented in Fig. S7. A first set of calculations was performed considering only dipolar interactions $\left(J_{\text {dip, }}\right.$ blue curve on Fig. S7). The resulting calculated $\chi_{\mathrm{M}} T$ curve reproduces the decrease upon cooling before reaching a minimum value of $51.87 \mathrm{~cm}^{3} \mathrm{~K} \mathrm{~mol}^{-1}$ at $18 \mathrm{~K}$. The $\chi_{\mathrm{M}} T$ product then increases upon further cooling to reach a value of $74.90 \mathrm{~cm}^{3} \mathrm{~K} \mathrm{~mol}^{-1}$ at $2 \mathrm{~K}$. This strong increase of the $\chi_{\mathrm{M}} T$ values observed in the low temperature range can be attributed to ferromagnetic dipolar interactions between the Dy(III) centers. Clearly, contrary to experimental results, the theoretical description of the low temperature behavior of $\mathbf{2}$ misses some important components that may be ascribed to exchange interactions. These exchange interactions are considered in subsequent calculations $\left(J_{\text {dip }}+J_{\text {ex }}\right)$ in order to balance the dipolar coupling contributions $\left(J_{\text {dip }}\right)$. These exchange parameters are fitted to obtain the best agreement with the experimental dc magnetic data (Fig. S7). The exchange interaction between the Dy2 and Dy2a centers $\left(J_{22 a}\right)$ is not considered in the calculations due to both the large Dy・・Dy distance and the lack of bridging ligands. A scan over the three different exchange values has been carried between -2.5 and 2.5 $\mathrm{cm}^{-1}$ (in the Ising model) and the set of $J_{12}, J_{11 \mathrm{a}}$ and $J_{21 \mathrm{a}}$ values corresponding to the best fit of the experimental $\chi_{\mathrm{M}} T$ data obtained for each compound are reported in Table 1.

Table 1. Best fitted exchange values $\left(\mathrm{cm}^{-1}\right.$, in the Ising model) for model complexes $\mathbf{1}^{\prime}$ and $\mathbf{2}^{\prime}$ within the scan range $[-2.5 ; 2.5] \mathrm{cm}^{-1}$.

\begin{tabular}{|c|c|c|c|}
\hline Complex & $J_{12}\left(\mathrm{~cm}^{-1}\right)$ & $J_{21 \mathrm{a}}\left(\mathrm{cm}^{-1}\right)$ & $J_{11 \mathrm{a}}\left(\mathrm{cm}^{-1}\right)$ \\
\hline $\mathbf{1}^{\prime}$ & -1.75 & -2.5 & 1 \\
\hline $\mathbf{2}^{\prime}$ & 0 & -2 & -2.25 \\
\hline
\end{tabular}

From complexes $\mathbf{1}$ to $\mathbf{2}$, efforts have been focused on the minute structural changes to explore the factors determining magnetic behaviors, and therefore fine-tuning the coordination environment located on the Dy1/1a center. The results show that 
the change of the coordination sphere around Dy $1 / 1 \mathrm{a}$, from ninecoordinated in complex $\mathbf{1}$ to eight-coordinated in $\mathbf{2}$, leads to a slight decrease of the antiferromagnetic contribution between the two nonequivalent Dy(III) centers $\left(J_{12}\right)$ while the exchange contribution between the two equivalent Dy(III) centers $\left(J_{11 a}\right)$ evolve from ferro to antiferromagnetic (Table 1). The low temperature $\chi_{\mathrm{M}} T$ behavior observed for $\mathbf{2}$ is explained by the overall antiferromagnetic intramolecular Dy••Dy exchange interactions that dominate the ferromagnetic dipolar contributions.

\section{Conclusions}

To summarize, the combination of experimental and theoretical techniques allowed the study of a new Dy(III) complex. On one hand the expertise in the synthesis allowed the selective change of coordination number and geometry on one of the magnetic center. On the other hand, the theoretical approach shed light on the effect of these structural modifications on the magnetic properties both on the local magnetic properties of the changeable site and on the intramolecular magnetic framework existing between the four magnetic Dy(III) ions. Indeed, the local change in the magnetic properties, on the Dy $1 / 1$ a sites, induced a modification of the magnetic interactions within the polynuclear unit. These modifications are reflected in the relaxation process occurring in the system allowing an enhancement of the latter from less than $2 \mathrm{~K}$ for $\mathbf{1}$ to $114 \mathrm{~K}$ for $\mathbf{2}$. This work offers hints in the modulation and optimization of SMM properties in dysprosium(III) polynuclear systems.

\section{Acknowledgements}

This work was supported by National Natural Science Foundation of China (Grants 21531007, 21525103 and 21521092), Natural Science Basic Research Plan in Shaanxi Province of China (Program No. 2018JQ2026), Scientific Research Program Funded by Shaanxi Provincial Education Department (Program No. 18JK0355). V.M. is thankful to ERC (project no. 725184) for fundings. B.L.G. thanks the French GENCI/IDRIS-CINES center for high-performance computing resources.

\section{Notes and references}

1 A. R. Rocha, V. M. García-suárez, S. W. Bailey, C. J. Lambert, J. Ferrer and S. Sanvito, Nat. Mater., 2005, 4, 335-339.

2 R. Vincent, S. Klyatskaya, M. Ruben, W. Wernsdorfer and F. Balestro, Nature, 2012, 488, 357-360.

3 S. Thiele, F. Balestro, R. Ballou, S. Klyatskaya, M. Ruben and W. Wernsdorfer, Science, 2014, 344, 1135-1138.

4 C. Cervetti, A. Rettori, M. G. Pini, A. Cornia, A. Repollé, F. Luis, M. Dressel, S. Rauschenbach, K. Kern, M. Burghard and L. Bogani, Nat. Mater., 2016, 15, 164-168.

5 E. Kiefl, M. Mannini, K. Bernot, X. Yi, A. Amato, T. Leviant, A. Magnani, T. Prokscha, A. Suter, R. Sessoli and Z. Salman, ACS Nano, 2016, 10, 5663-5669.

6 D. Gatteschi, R. Sessoli and J. Villain, Molecular Nanomagnets, Oxford University Press, Oxford, 2006.
7 F. S. Guo, B. M. Day, Y. C. Chen, M. L. Tong, A. Mansikkamäki and R. A. Layfield, Science, 2018, DOI: 10.1126/science. aav0652.

8 K. R. McClain, C. A. Gould, K. Chakarawet, S. J. Teat, T. J. Groshens, J. R. Long and B. G. Harvey, Chem. Sci., 2018, 9, 8492-8503.

9 J. D. Rinehart and J. R. Long, Chem. Sci., 2011, 2, 2078-2085.

10 N. F. Chilton, D. Collison, E. J. L. Mclnnes, R. E. P. Winpenny and A. Soncini, Nat. Commun., 2013, 4, 2551.

11 P. Zhang, Y. N. Guo and J. K. Tang, Coord. Chem. Rev., 2013, 257, 1728-1763.

12 J. L. Liu, Y. C. Chen and M. L. Tong, Chem. Soc. Rev., 2018, 47, 2431-2453.

13 S. D. Jiang, B. W. Wang, G. Su, Z. M. Wang and S. Gao, Angew. Chem. Int. Ed., 2010, 49, 7448-7451.

14 G. J. Chen, Y. N. Guo, J. L. Tian, J. Tang, W. Gu, X. Liu, S. P. Yan, P. Cheng and D. Z. Liao, Chem.-Eur. J., 2012, 18, 2484-2487.

15 G. Cucinotta, M. Perfetti, J. Luzon, M. Etienne, P. E. Car, A. Caneschi, G. Calvez, K. Bernot and R. Sessoli, Angew. Chem., Int. Ed., 2012, 51, 1606-1610.

16 P. Zhang, L. Zhang, C. Wang, S. Xue, S. Y. Lin and J. K. Tang, J. Am. Chem. Soc., 2014, 136, 4484-4487.

17 W. B. Sun, P. F. Yan, S. D. Jiang, B. W. Wang, Y. Q. Zhang, H. F. Li, P. Chen, Z. M. Wang and S. Gao, Chem. Sci., 2016, 7, 684-691.

18 J. F. Wu, J. L. Jung, P. Zhang, H. X. Zhang, J. K. Tang and B. Le Guennic, Chem. Sci., 2016, 7, 3632-3639.

19 Y. C. Chen, J. L. Liu, L. Ungur, J. Liu, Q. W. Li, L. F. Wang, Z. P. Ni, L. F. Chibotaru, X. M. Chen and M. L. Tong, J. Am. Chem. Soc., 2016, 138, 2829-2837.

20 J. Liu, Y. C. Chen, J. L. Liu, V. Vieru, L. Ungur, J. H. Jia, L. F. Chibotaru, Y. Lan, W. Wernsdorfer, S. Gao, X. M. Chen and M. L. Tong, J. Am. Chem. Soc., 2016, 138, 5441-5450.

21 Y. S. Ding, N. F. Chilton, R. E. P. Winpenny and Y. Z. Zheng, Angew. Chem. Int. Ed., 2016, 55, 16071-16074.

22 S. K. Gupta, T. Rajeshkumar, G. Rajaraman and R. Murugavel, Chem. Sci., 2016, 7, 5181-5191.

23 T. Gupta and G. Rajaraman, Chem. Commun., 2016, 52, 89729008.

24 A. B. Canaj, M. K. Singh, C. Wilson, G. Rajaraman and M. Murrie, Chem. Commun., 2018, 54, 8273-8276.

25 I. J. Hewitt, J. K. Tang, N. T. Madhu, C. E. Anson, Y. H. Lan, J. Luzon, M. Etienne, R. Sessoli and A. K. Powell, Angew. Chem. Int. Ed., 2010, 49, 6352-6356.

26 E. M. Pineda, N. F. Chilton, R. Marx, M. Dörfel, D. O. Sells, P. Neugebauer, S. D. Jiang, D. Collison, J. V. Slageren, E. J. L. Mclnnes and R. E. P. Winpenny, Nat. Commun., 2014, 5, 5243.

27 J. Xiong, H. Y. Ding, Y. S. Meng, C. Gao, X. J. Zhang, Z. S. Meng, Y. Q. Zhang, W. Shi, B. W. Wang and S. Gao, Chem. Sci., 2017, 8, 1288-1294.

28 M. J. Giansiracusa, E. Moreno-Pineda, R. Hussain, R. Marx, M. M. Prada, P. Neugebauer, S. Al-Badran, D. Collison, F. Tuna, J. Slageren, S. Carretta, T. Guidi, E. J. L. Mclnnes, R. E. P. Winpenny and N. F. Chilton, J. Am. Chem. Soc., 2018, 140, 2504-2513.

29 Y. N. Guo, G. F. Xu, P. Gamez, L. Zhao, S. Y. Lin, R. P. Deng, J. K. Tang and H. J. Zhang, J. Am. Chem. Soc., 2010, 132, 8538-8539.

30 Y. M. Song, F. Luo, M. B. Luo, Z. W. Liao, G. M. Sun, X. Z. Tian, Y. Zhu, Z. J. Yuan, S. J. Liu, W. Y. Xu and X. F. Feng, Chem. Commun., 2012, 48, 1006-1008.

31 R. J. Blagg, L. Ungur, F. Tuna, J. Speak, P. Comar, D. Collison, W. Wernsdorfer, E. J. L. Mclnnes, L. F. Chibotaru and R. E. P. Winpenny, Nat. Chem., 2013, 5, 673-678.

32 Y. L. Wang, C. B. Han, Y. Q. Zhang, Q.Y. Liu, C. M. Liu and S. G. Yin, Inorg. Chem., 2016, 55, 5578-5584. 
33 K. Zhang, D. Liu, V. Vieru, L. Hou, B. Cui, F. S. Guo, L. F. Chibotaru and Y. Y. Wang, Dalton Trans., 2017, 46, 638-642.

34 K. Zhang, V. Montigaud, O. Cador, G. P. Li, B. Le Guennic, J. K. Tang and Y. Y. Wang, Inorg. Chem., 2018, 57, 8550-8557.

35 Y. N. Guo, G. F. Xu, W. Wernsdorfer, L. Ungur, Y. Guo, J. K. Tang, H. J. Zhang, L. F. Chibotaru and A. K. Powell, J. Am. Chem. Soc., 2011, 133, 11948-11951.

36 J. D. Rinehart, M. Fang, W. J. Evans and J. R. Long, Nat. Chem., 2011, 3, 538-542.

37 F. Yang, Q. Zhou, G. Zeng, G. H. Li, L. Gao, Z. Shi and S. H. Feng, Dalton Trans., 2014, 43, 1238-1245.

38 Y. B. Lu, X. M. Jiang, S. D. Zhu, Z. Y. Du, C. M. Liu, Y. R. Xie and L. X. Liu, Inorg. Chem., 2016, 55, 3738-3749.

39 L. Zhang, Y. Q. Zhang, P. Zhang, L. Zhao, M. Guo and J. K. Tang, Inorg. Chem., 2017, 56, 7882-7889.

40 G. F. Garcia, D. Guettas, V. Montigaud, P. Larini, R. Sessoli, F. Totti, O. Cador, G. Pilet and B. Le Guennic, Angew. Chem. Int. Ed., 2018, 52, 17335-17339.

41 H. S. Ke, L. Zhao, Y. Guo and J. K. Tang, Inorg. Chem., 2012, 51, 2699-2705.

42 G. M. Sheldrick, SHELXS-2014, Program for Crystal Structure Solution, University of Göttingen, 2014.

43 M. Llunell, D. Casanova, J. Cirera, P. Alemany and S. Alvarez, Shape v. 2.0, Universitat de Barcelona, Barcelona, 2010.

44 O. Kahn, Molecular Magnetism, Wiley-VCH, New York, 1993.

45 K. Zhang, C. Yuan, F. S. Guo, Y. Q. Zhang and Y. Y. Wang, Dalton Trans., 2017, 46, 186-192.

46 K. Zhang, F. S. Guo and Y. Y. Wang, Dalton Trans., 2017, 46, 1753-1756.

47 D. Gatteschi, R. Sessoli and J. Vallain, Molecular Nanomagnets, Oxford University Press, New York, 2006.

48 M. Hagiwara, J. Magn. Magn. Mater., 1998, 177-181, 89-90.

49 L. L. Zhao, Y. N. Guo, G. M. Yu, Y. Guo, J. K. Tang and Y. H. Li, Chem. Commun., 2011, 47, 8659-8661.

50 F. Aquilante, J. Autschbach, R. K. Carlson, L. F. Chibotaru, M. G. Delcey, L. De Vico, I. F. Galván, N. Ferré, L. M. Frutos, L. Gagliardi, M. Garavelli, A. Giussani, C. E. Hoyer, G. L. Manni, H. Lischka, D. X. Ma, P. Malmqvist, T. Müller, A. Nenov, M. Olivucci, T. B. Pedersen, D. L. Peng, F. Plasser, B. Pritchard, M. Reiher, I. Rivalta, I. Schapiro, J. Segarra-Martí, M. Stenrup D. G. Truhlar, L. Ungur, A. Valentini, S. Vancoillie, V. Veryazov, V. P. Vysotskiy, O. Weingart, F. Zapata and R. Lindh, J. Comput. Chem., 2016, 37, 506-541. 